\title{
Silicon nanowire photodetector enhanced by a bow-tie antenna
}

\author{
Gordana Klaric Felic ${ }^{1}$, Feras Al-Dirini ${ }^{2,1}$, Faruque M. Hossain ${ }^{1,2}$, Thanh Cong Nguyen ${ }^{2,1}$, Efstratios \\ Skafidas ${ }^{2,1}$
}

\author{
${ }^{1}$ National ICT Australia, The University of Melbourne, Parkville VIC 3010, Australia \\ ${ }^{2}$ The University of Melbourne, Electrical and Electronic Engineering, Parkville VIC 3010, Australia \\ *corresponding authors, E-mails: gordana.felic@nicta.com.au, fhossain@unimelb.edu.au
}

\begin{abstract}
Properties of surface plasmon polaritons (SPP) can be exploited for the minituarisation of photonic circuits below the optical wavelength scale. Smaller and more sensitive photodetectors can be made by using sub-wavelength semiconductor elements such as germanium or silicon nanowires in combination with nanometer-scale antennas. Proposed nanowire photodetector enables on-chip optical sensing applications with increased sensitivity and reduced size.
\end{abstract}

\section{Introduction}

Excellent electronic transport properties of silicon nanowires can be exploited for biomolecule detection in the $\mathrm{dc}$ and frequency dependent detection systems [1]. Germanium and silicon-based nanowire devices can absorb and transduce light to photocurrent [2-4]. The addition of optical antennas in the nanowire device enhances photodetection by concentrating radiation into a semiconductor nanowire. This is due to the fact that optical nano-antennas can increase the absorption cross-section and provides better signal-to-noise ratio. They also may enable manipulating optical fields at nanometer-scale, and thus help in enhancing the efficiency of nano-scale photodetectors. This property of optical antenna comes from the unique optical behavior of metals that enable collective electron excitations, known as surface plasmons [5]. In this paper we present and discuss how a bow-tie dipole antenna can be exploited to confine the radiated power into a silicon nanowire. Using principle-of-field enhancement by metal antenna structures a subwavelength metal-semiconductormetal detector can be built [2].

\section{Device construction}

The silicon nanowire device is patterned on a silicon-oninsulator (SOI) wafer in order to maintain compatibility with planner CMOS fabrication process. The silicon dioxide insulator layer is $150 \mathrm{~nm}$ thick and the top silicon device has a thickness of $50 \mathrm{~nm}$. Figure 1(a) shows a perspective view of the proposed device. Silicon nanowire is shown as traced through the gap of printed gold antennas (bow-tie and/or Hertz-dipole). Gold pads at two ends of the nanowire (to form electrical contacts) and bow-tie antenna may be patterned and deposited using one process step. The length and diameter of the Si nanowire are considered to be 500 $\mathrm{nm}$ and $50 \mathrm{~nm}$ respectively. The total length of our designed antenna is $380 \mathrm{~nm}$ and the gap between the metal arms is $100 \mathrm{~nm}$.

\section{Results and discussion}

To quantify the enhancement of light absorption, simulation has been performed using the CST (Computer Simulation Technology) Microwave Studio, Time Domain (TD) Solver [6]. The simulation model is based on the previously constructed nanowire device without antenna [2]. The computation of power flow on the surface of the nanowire is obtained by integration of the power density over the nanowire surface.

\subsection{Power Flow Computation}

According to the conservation theorem [7], the power flowing into the circuit is equal to the rate of change of the energy stored and the rate of energy dissipation. In theoretical generalization, the energy is distributed through space with an energy density $\mathrm{W}\left(\mathrm{J} / \mathrm{m}^{3}\right)$, and the power is dissipated at a local rate of dissipation per unit volume $\mathrm{P}_{\mathrm{d}}$ $\left(\mathrm{W} / \mathrm{m}^{3}\right)$. As the power on the nanowire flows with a density $\mathbf{S}\left(\mathrm{W} / \mathrm{m}^{2}\right)$, and direction vector $\mathbf{a}$, the power crossing the nanowire surface, $S_{a}$ is given in [7] by

$$
\oint_{S_{a}} \mathbf{S} d \mathbf{a}
$$

With these field-theoretical generalizations, the power flowing into the nanowire volume $\mathrm{V}$, enclosed by the surface $\mathrm{S}$ is given in [7] by

$$
-\oint_{S_{a}} \mathbf{S} d \mathbf{a}=\frac{d}{d t} \int_{V} W d V+\int_{V} P_{a} d V
$$

where the minus sign indicates that the term on the left is the power flowing into the volume. According to the right hand side of the equation, input power is equal to the rate of increase of the total energy stored plus the power dissipation. The total energy is expressed as an integral over the volume of an energy density, $W$ and the total power dissipation is the integral over the volume of a power dissipation density $P_{d}$. 


\subsection{Optical Bow-Tie Antenna}

When a bow-tie antenna is illuminated with electromagnetic radiation, currents are induced in the arms of the antenna and guided towards the open circuit terminal (gap) region. The accumulated charge at the open terminals results in displacement current across the gap (field enhancement region). Considering, a fundamental resonance, the effective wavelength of the bow-tie antenna depends on the length $\mathrm{L}$ and the effective index of the localized SPP along the bowtie antenna gap, $\mathrm{n}_{\text {eff }}[8]$

$$
\lambda \approx 2 \int_{-L / 2}^{L / 2} n_{\text {eff }}(x) d x+\delta
$$

where is the offset due to the antenna gap and reflections. The above equation is a simple linear scaling law for eff where $\mathrm{n}_{\text {eff }}$ has a dimension of antenna length depending on the geometry and dielectric properties of the metal at optical frequencies (plasma wavelength).

\subsection{Results}

When the photodetector with the antenna is illuminated with a plane wave in perpendicular direction to the nanowire (along the gap of the bow-tie antenna), the electric field intensity will increase. This will drive the increased light absorption in the nanowire. Power flow (absorption) distribution profile for the nanowire with bow-tie antenna and dipole antenna are given in Figure 2. The near-field and power flow distribution of the detector have been calculated for the plane wave excitations at wavelengths from $300 \mathrm{~nm}$ to $1.6 \mu \mathrm{m}$.

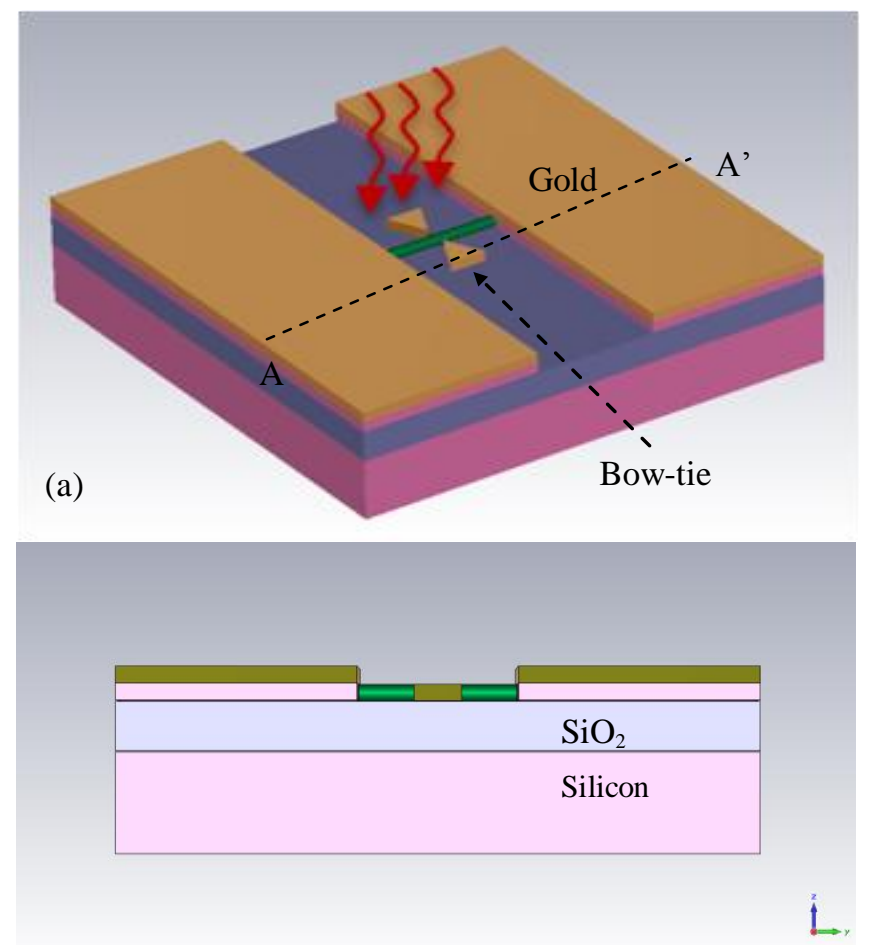

(b)

Figure 1: Schematic illustration of the semiconductor nanowire device used for photo detection. (a) Perspective view (b) Cross-sectional view of the device (AA').
The ability of a bow-tie antenna to concentrate radiation in the silicon element (absorption enhancement) is illustrated in Figure 3. The plane wave polarised in the bow-tie direction was incident from the top of the nanowire device. The structure with bow-tie antenna resonates at a wavelength of $850 \mathrm{~nm}$ with highest electric field in the gap region. In order to compare the effect of the antennas (bow-tie and Hertz dipole) on light absorption enhancement, power density is integrated on the face of the wire (absorbed power) for the given range of wavelengths. The power absorption of the system with both antennas is then compared with the power absorption of the silicon wire. The comparison between the power absorption for a half-wave Herzian dipole antenna and bow-tie antenna is given in Figure 4. It shows that the bow-tie antenna enhances the power absorption for the entire range of wavelength $(300-1600 \mathrm{~nm})$ and that the absorption enhancement is greater than the absorption enhancement of the half-wave Hertz dipole antenna. These results indicate the ability of bow-tie antennas of reducing semiconductor detector's area many-fold and thus the enhancement of sensitivity at several orders of magnitude.
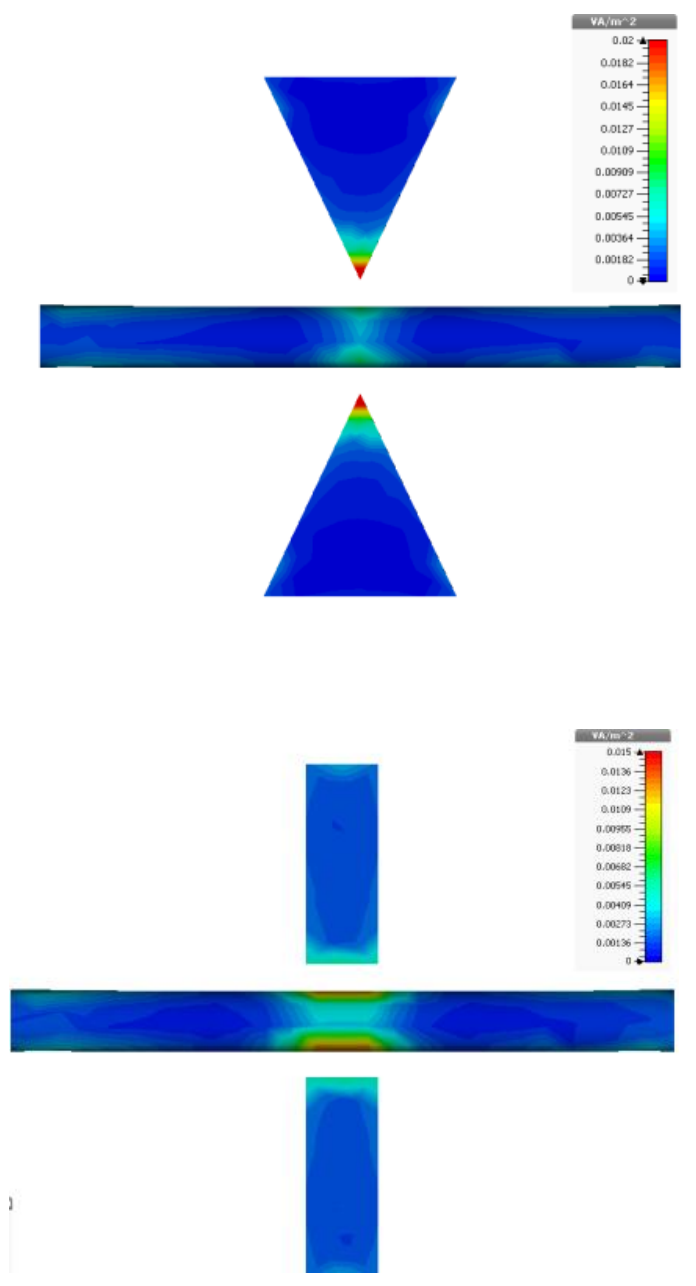

Figure 2: The distribution profile of power flow $\left(\mathrm{VA} / \mathrm{m}^{2}\right)$ for the nanowire system with bow-tie and dipole antenna (antenna length, $380 \mathrm{~nm}$, silicon wire diameter, $50 \mathrm{~nm}$, at 850 $\mathrm{nm})$. 


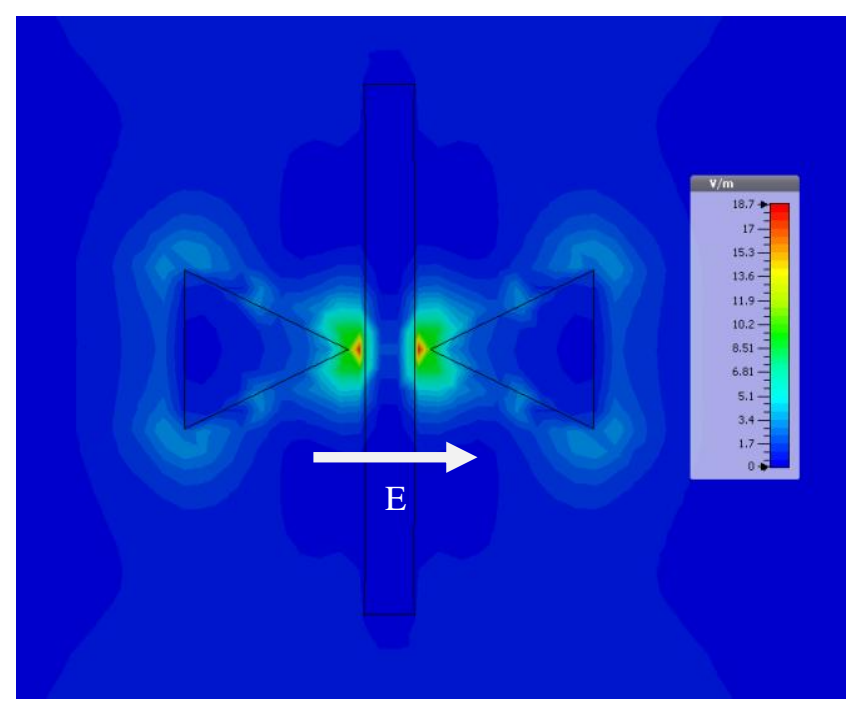

Figure 3: The distribution profile of electric field for the nanowire detection system with bow-tie antenna at wavelength, $=850 \mathrm{~nm}$.

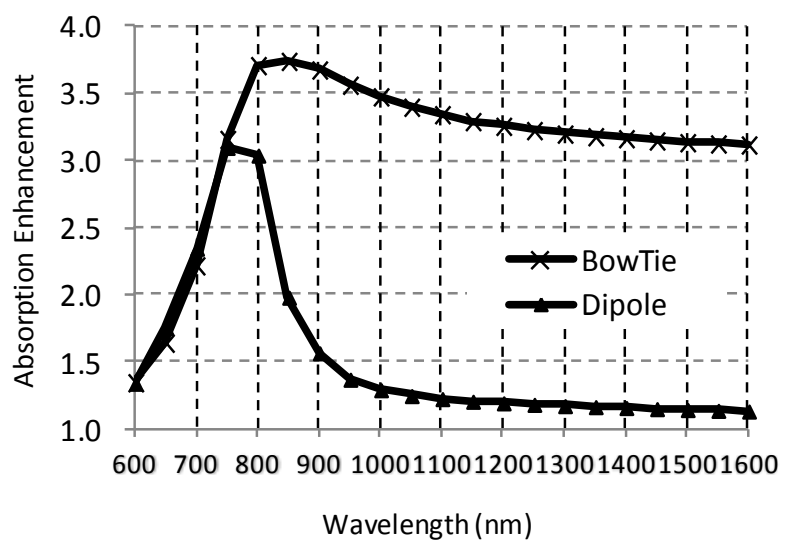

Figure 4: Spectra of light absorption enhancement for the nanowire system with bow-tie antenna and dipole antenna (wire length: $500 \mathrm{~nm}$, diameter: $50 \mathrm{~nm}$ ).

\section{Conclusion}

In this paper we have shown that the inclusion of bow-tie antenna can increase the ability to absorb light for a wide range of wavelength and can provide the enhancement of sensitivity of the semiconductor photodetector without involving far-field radiation. Thus the designed nano-wire based nano-antenna system holds promise for both highly sensitive photodetection and photovoltaics applications.

\section{Acknowledgements}

NICTA is funded by the Australian Government as represented by the Department of Broadband, Communications and the Digital Economy and the Australian Research Council through the ICT Centre of Excellence program.

\section{References}

[1] Nguyen T.C, Qiu W. Z. and Skafidas E. "Functionalised Nanowire-Based Antigen Detection Using FrequencyBased Signals, IEEE Transactions on Biomedical Engineering, VOL. 59, NO. 1, January 2012.

[2] Tang, L. et al. "Nanometre-scale germnium photodetector enhanced by a near-infrared dipole antenna," Nature Photonics, VOL. 2, 226-229, 2008.

[3] Fan P, Chettiar U. K., Cao L, Afsinmanesh F., Engheta N. and Brongersma M. L., "An invisible metalsemiconductor photodetector," Nature Photonics, VOL. 6, 380-385, 2012.

[4] Pengyu F., Huang C.Y., Linyou C. and Brongersma M. L., "Redesigning Photodetector Electrodes as an Optical Antenna," Nano. Lett. 2013, 13 (2), pp 392-396.

[5] J.A. Schuller et al., "Plasmonics for extreme light concentration and manipulation", Nature Materials, VOL 9, 193-204, 2010.

[6] http://www.cst.com/

[7] Haus, Hermann A., and James R. Melcher. Electromagnetic Fields and Energy. Englewood Cliffs, NJ: Prentice-Hall, 1989

[8] L. Novotny, "Effective Wavelength Scaling for Optical Antennas," Physical Review Letters, PRL 98, 266802 (2007) 


\section{University Library}

\section{- M M N E R VA A gateway to Melbourne's research publications}

Minerva Access is the Institutional Repository of The University of Melbourne

Author/s:

Felic, GK;Al-Dirini, F;Hossain, FM;Thanh, CN;Skafidas, E

Title:

Silicon nanowire photodetector enhanced by a bow-tie antenna

Date:

2014-05-01

Citation:

Felic, G. K., Al-Dirini, F., Hossain, F. M., Thanh, C. N. \& Skafidas, E. (2014). Silicon nanowire photodetector enhanced by a bow-tie antenna. APPLIED PHYSICS A-MATERIALS SCIENCE \& PROCESSING, 115 (2), pp.491-493. https://doi.org/10.1007/s00339-013-8070-z.

Persistent Link:

http://hdl.handle.net/11343/282713 